\title{
Anisotropic Magnetocaloric Effect in Nanostructured Magnetic Clusters
}

\author{
X.X. Zhang, * H. L. Wei, Z. Q. Zhang, and Lingyun Zhang \\ Department of Physics and Institute of Nano Science and Technology (INST), The Hong Kong University \\ of Science and Technology, Clear Water Bay, Kowloon, Hong Kong, China
}

(Received 28 August 2000; revised manuscript received 6 July 2001; published 25 September 2001)

We report the first experimental observation of anisotropic magnetocaloric effect (MCE) in the $\mathrm{Fe}_{8}$ clusters. It is found that the magnetic anisotropy plays a very important role in the determination of the magnetocaloric effect. The maximum and minimum MCE's are observed when the applied magnetic fields are parallel and perpendicular to the easy axis, respectively. The quantum spin Hamiltonian of a $\mathrm{Fe}_{8}$ cluster is used to calculate the partition function and the magnetization in a range of temperature and magnetic field. Excellent quantitative agreement between the experimental data and calculation is observed.

\section{DOI: $10.1103 /$ PhysRevLett.87.157203}

It is well known that the magnetocaloric effect (MCE) has been useful in achieving very low temperatures ( $\mathrm{mK}$ range) [1]. Up to now, most of MCE applications still remain in the low temperature range $(<20 \mathrm{~K})$ by using paramagnetic salts, e.g., $\mathrm{Gd}_{3} \mathrm{Ga}_{3} \mathrm{O}_{12}$ [2]. For the MCE applications at high temperatures, different types of materials must be used [3-6]. The MCE in the superparamagnetic nanostructured materials has been recently studied by Shull and co-workers [7-10]. The results indicate that at higher temperatures the MCE is larger in the superparamagnetic nanostructures composites than in the pure paramagnetic materials. This makes the nanostructured composites appear to be very attractive candidates for the magnetic refrigeration in a very big temperature span. This enhancement was discussed by Shull et al. in considering a superparamagnetic system consisting of monosized and noninteracting magnetic clusters uniformly dispersed in a nonmagnetic media $[7,10]$.

It is well known that most magnetic nanoparticles or clusters exhibit a quite strong uniaxial magnetic anisotropy due to shape, stress, or surface effects [11], which governs the magnetic behavior of the particles $[11,12]$. Therefore, it is essential to understand the effect of the magnetic anisotropy in the MCE, particularly for the superparamagnetic materials. However, not much effort has been devoted to it. The reason could be that the materials used for magnetic refrigerants are either the ideal paramagnetic salts [2] or soft magnetic materials [3-6] and therefore the effect of magnetic anisotropy is negligible. Theoretically, the anisotropic MCE was studied by using the Monte Carlo simulation for bulk ferromagnetic materials (or interacting magnetic clusters) with a uniaxial anisotropy in the vicinity of the Curie temperature [13]. Experimentally, a clean study of the anisotropic effect is generally hindered by the distribution of sizes and/or interactions. In this Letter, we report the anisotropy effect on the MCE in $\mathrm{Fe}_{8}$ magnetic molecular crystals. With monosized clusters and well-defined anisotropic properties, this system offers, for the first time, a definite confirmation
PACS numbers: 75.30.Gw, 75.30.Sg, 75.50.Tt, 75.50.Xx

of the anisotropic MCE. It is shown that the magnetic anisotropy is important for both the qualitative and quantitative aspects of the MCE.

The cluster $\mathrm{Fe}_{8}$ with the formula $\left[(\operatorname{tacn})_{6} \mathrm{Fe}_{8} \mathrm{O}_{2}\right.$ $\left.(\mathrm{OH})_{12}\right]_{8+}$, where tacn is the organic ligand trizacyclononane, has an approximate $D_{2}$ symmetry [14]. The magnetic characteristics of the clusters, studied by using EPR, ac, and dc magnetic techniques [15-17], suggest that the $\mathrm{Fe}_{8}$ clusters have a ground state with spin $S=10$ [18]. The $\mathrm{Fe}_{8}$ clusters can crystallize into single crystals $\mathrm{mm}$ in size. The crystal cell parameters are $a=10.609(7), b=14.15(2), c=15.002(9) \AA, \alpha=$ $89.45(9)^{\circ}, \beta=10.03(5)^{\circ}$, and $\gamma=109.42(9)^{\circ}[14,19]$. The magnetic interactions among the clusters are therefore negligible for the large enough lattice parameters. The magnetic anisotropy field of the clusters was found to be $\sim 5 \mathrm{~T}$ from the aligned samples [17]. The molecular crystals of $\mathrm{Fe}_{8}$ can therefore be considered to be a collection of a huge number of identical, aligned, noninteracting single domain particles with a moment of $20 \mu_{B}$. This material, as well as another magnetic molecular crystal $\mathrm{Mn}_{12}$, has been studied very intensively, both experimentally and theoretically, in the context of resonant spin tunneling [16,17,19-23]. The $\mathrm{Fe}_{8}$ and $\mathrm{Mn}_{12}$ crystals constitute a model system for testing many theoretical predictions on the macroscopic tunneling. Recently the time-dependent magnetocaloric effect has been observed in $\mathrm{Mn}_{12}$ and $\mathrm{Fe}_{8}$ clusters at low temperatures, which was ascribed to the relaxation effect [24]. We concentrate here on the effect of magnetic anisotropy on the MCE in a high temperature regime, where the sample is always in the thermodynamic equilibrium states.

The $\mathrm{Fe}_{8}$ crystallites of an average size of $0.2 \times 0.1 \times$ $0.1 \mathrm{~mm}$ used here are the same as those for the study of resonant tunneling [17]. The aligned sample is a solid cylinder with a diameter of $3 \mathrm{~mm}$ and a length of $6 \mathrm{~mm}$, in which the easy axis ( $c$ axes) of the $\mathrm{Fe}_{8}$ clusters is along the axis of the column [17]. By changing the angle $\theta$ between the $c$ axis and applied field, it is possible to study the anisotropy effects. 
As reported in Ref. [17], the clusters behave superparamagnetically down to $1.8 \mathrm{~K}$, in agreement with a recent study in the $\mathrm{mK}$ regime [24]. The field-dependent magnetizations were measured at 1.8 and $2.5 \mathrm{~K}$ for different values of $\theta$. It is found that the behavior of the magnetization depends strongly on $\theta$. At about $5 \mathrm{~T}$, the magnetization curve at $\theta=90^{\circ}$ appears to be saturated, indicating a $5 \mathrm{~T}$ anisotropy field [17]. The quality of alignment of the crystallites can be determined by using the magnetization curve obtained at $1.8 \mathrm{~K}$ with the field parallel to the easy axis. The ratio of the magnetization $M(5 \mathrm{~T})$ to the saturation magnetization $M(0), M(0) / M(5 \mathrm{~T}) \approx 0.99$ suggests that the misalignment of the easy axes of the crystallites with respect to the applied magnetic field is no more than $1^{\circ}$ $[15,17]$.

The isothermal magnetization was measured with a field step of $500 \mathrm{Oe}$ in the field range of $0-4 \mathrm{~T}$ for different $\theta$ 's. It is adequate to consider the magnetization curves to be isothermal for a sufficiently low sweeping rate of the magnetic field $[5,6,25]$. The magnetic entropy change, $-\Delta S_{M}$, can then be calculated by using the magnetization data and the numerical formula [8]

$$
-\Delta S_{M}=\sum_{i} \frac{1}{T_{i+1}-T_{i}}\left(M_{i}-M_{i+1}\right) \Delta H_{i},
$$

where $M_{i}$ and $M_{i+1}$ are the magnetization values measured at the temperatures $T_{i}$ and $T_{i+1}$, respectively, in a field $H$. Figure 1 shows the isothermal magnetization curves measured with the applied field parallel to the easy axis and "hard axis" [26]. It is evident that in these two cases the curves show very different behaviors, particularly at low temperatures. In Fig. 2, the $-\Delta S_{M}$ measured for a field change from 0 to $3 \mathrm{~T}$ is plotted as a function of temperature. The most interesting feature in this figure is that the MCE depends strongly on the angle between the applied magnetic field and the easy axis of the sample

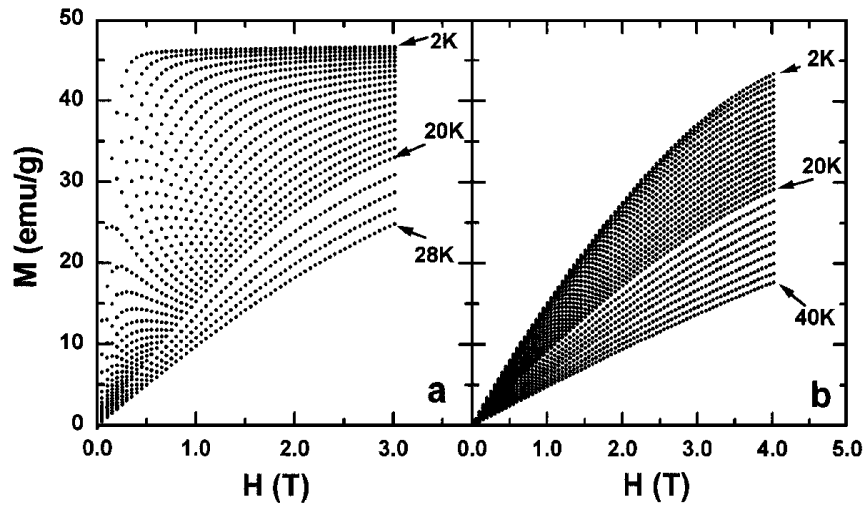

FIG. 1. The isothermal magnetization measured at different temperatures. (a) The applied field parallel to the easy axis $(\theta=0)$; the temperature steps are, respectively, 1 and $2 \mathrm{~K}$ for the temperature ranges $2-20 \mathrm{~K}$ and $20-28 \mathrm{~K}$. (b) The applied field parallel to the hard axis $\left(\theta=90^{\circ}\right)$ [26]; the temperature steps are 1 and $2 \mathrm{~K}$ for the temperature ranges $2-20 \mathrm{~K}$ and 20-40 K, respectively. i.e., anisotropic MCE. Examination of the data in Fig. 2 reveals that the highest and lowest MCE's appear, respectively, when the magnetic field is parallel and perpendicular to the easy axis, which is in agreement with the Monte Carlo simulation results [13]. Another interesting feature is that the maxima in $-\Delta S_{M}(T)$ is much higher than the blocking temperature of the $\mathrm{Fe}_{8}$ clusters obtained in the zero field cooled and field cooled magnetization curves [24]. The anisotropic MCE may be understood qualitatively by examining the definition of $-\Delta S_{M}(H, T)$ for a magnetic field change $\Delta H=H_{2}-H_{1}$,

$$
\begin{aligned}
\Delta S_{M} & =S_{M}\left(H_{2}, T\right)-S_{M}\left(H_{1}, T\right) \\
& =\int_{H_{1}}^{H_{2}}\left(\frac{\partial M(H, T)}{\partial T}\right)_{H} d H .
\end{aligned}
$$

When a field, smaller than the anisotropy field, is applied at an angle $\theta(\neq 0)$ the magnetization $M$ is smaller than when the field is applied parallel to the easy axis. If the magnetization has the same temperature dependencies in both cases, the larger the magnetization, the larger the term $(\partial M / \partial T)_{H}$. Actually, $M$ decreases more slowly at $\theta \neq 0$ than at $\theta=0$, which diminishes further the term $(\partial M / \partial T)_{H}$ for $\theta \neq 0$, and, consequently, $-\Delta S_{M}$. In the inset of Fig. 2, we plot the temperature-dependent magnetization obtained in a field of $1 \mathrm{kOe}$ applied along and perpendicular to the easy axis, which confirms our expectation for $T<40 \mathrm{~K}$. Above $40 \mathrm{~K}$, the characteristics of the clusters may no longer exist $[14,15]$. In addition to the size difference in $-\Delta S_{M}$ for different $\theta$ 's, the behavior of the curves in Fig. 2 is not the same, which may be due to the different temperature dependencies of the magnetization.

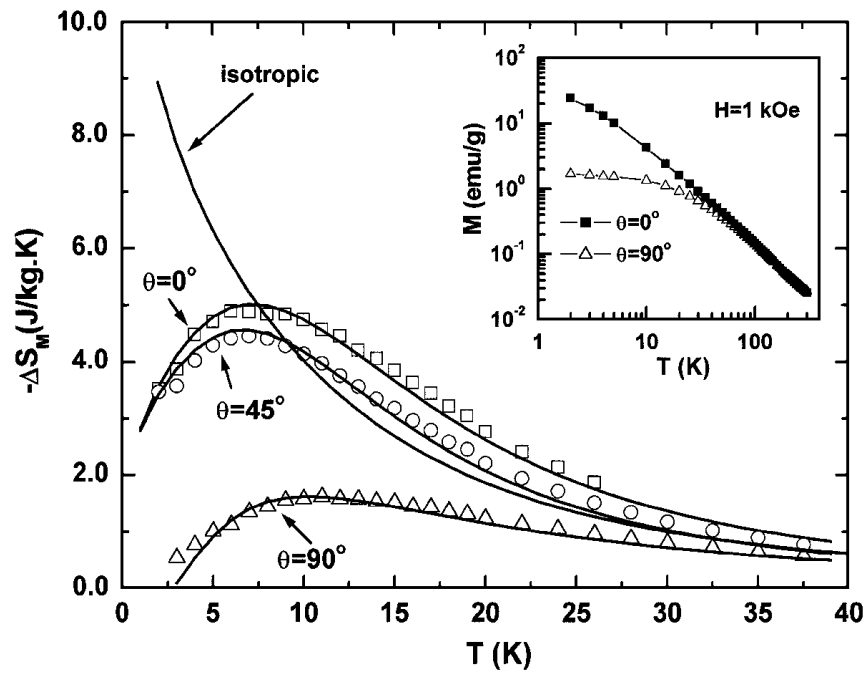

FIG. 2. The temperature dependence of the magnetic entropy change of $\mathrm{Fe}_{8}$ aligned sample (different angles) (symbols) and numerical simulation for the isotropic clusters. The solid lines are the calculation results. The field is changed from 0 to $3 \mathrm{~T}$. Inset: temperature-dependent magnetization obtained in a $1 \mathrm{kOe}$ magnetic field, for $\theta=0$ and $90^{\circ}$. 
To gain a deeper understanding of the anisotropy effect on the magnetic entropy change, we calculated the isothermal magnetization curves for $\mathrm{Fe}_{8}$ clusters at different field orientations relative to the easy axis. Similar calculations have been performed for the isotropic clusters with a moment $\mu=20 \mu_{B}$, the same as that of $\mathrm{Fe}_{8}$ clusters. Here, the isotropic particle system was supposed to possess all the characteristics of the $\mathrm{Fe}_{8}$ crystal, except for the magnetic anisotropy. For $\mathrm{Fe}_{8}$ clusters, the partition function and the magnetization curves are calculated by solving the eigenvalue problem of the following first order approximation of the spin Hamiltonian for a $\mathrm{Fe}_{8}$ cluster with $S=10$ [26]:

$$
H=-D S_{z}^{2}-g \mu_{B} h\left(\sin \theta S_{x}+\cos \theta S_{z}\right),
$$

where $g=2$ and $D=0.27 \mathrm{~K}$ is the isotropy energy. Here, we have taken $z$ as the easy axis and assumed that the magnetic field $\vec{h}$ is in the $x-z$ plane with an angle $\theta$ relative to the $z$ axis. $S_{x}$ and $S_{z}$ are the spin operators along the $x$ and $z$ axes, respectively. The matrix form of Eq. (3) can be obtained by choosing a basis that diagonalizes $S_{z}$, i.e., $S_{z}|S, m\rangle=m|S, m\rangle$, with $m=0, \pm 1, \pm 2, \ldots, \pm 10$. In this representation, the matrix elements of $S_{x}$ can be obtained by using the relation $S_{x}=\left(S_{+}+S_{-}\right) / 2$, where $S_{+}$and $S_{-}$are, respectively, the raising and lowering operators, yielding $\left(S_{x}\right)_{n m}=\left\langle n\left|S_{x}\right| m\right\rangle=\left[\sqrt{S(S+1)-m(m+1)} \delta_{n, m+1}+\right.$ $\left.\sqrt{S(S+1)-m(m-1)} \delta_{n, m-1}\right] / 2$. We diagonalize Eq. (3) numerically and find a set of eigenenergies, $\varepsilon(i)$, and eigenfunctions, $\left|\psi_{i}\right\rangle=\sum_{n} a_{n i}|n\rangle$. The partition function and the magnetization are evaluated by using the relations $Z=\sum_{i} \exp \left[-\varepsilon(i) / k_{b} T\right]$ and $\vec{M}=$ $g \mu_{B}\left\{\sum_{i}\left\langle\psi_{i}|\vec{S}| \psi_{i}\right\rangle \exp \left[-\varepsilon(i) / k_{b} T\right]\right\} / Z$ with $\left\langle\psi_{i}\left|S_{x}\right| \psi_{i}\right\rangle=$ $\sum_{n, m} a_{n i}^{*}\left(S_{x}\right)_{n m} a_{m i}$ and $\left\langle\psi_{i}\left|S_{z}\right| \psi_{i}\right\rangle=\sum_{n} n\left|a_{n i}\right|^{2}$. The measured magnetization along the applied field becomes $M(T, h)=\vec{M} \cdot \vec{h} /|\vec{h}|=M_{x} \sin \theta+M_{z} \cos \theta$. Figure 3 shows the calculated isothermal magnetization curves for $\theta=0$ and $45^{\circ}$ at several selected temperatures for comparison with the experimental data. Excellent quantitative agreement is seen for all $\theta$ 's up to $18 \mathrm{~K}$. Above $18 \mathrm{~K}$, the calculated data begin to deviate from the experimental

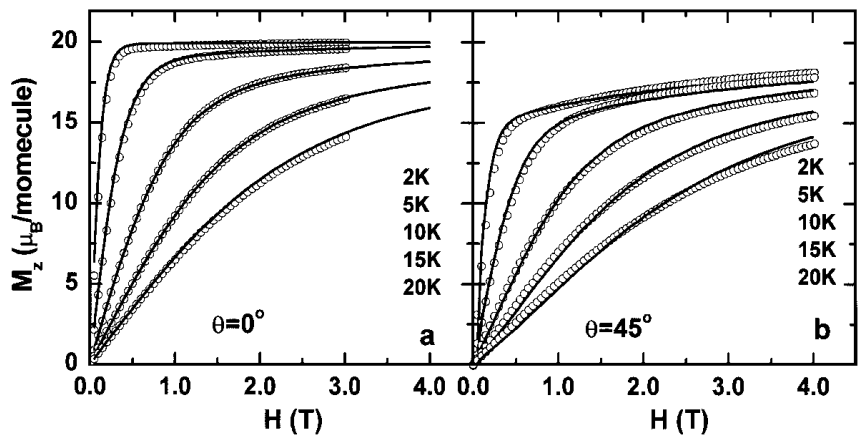

FIG. 3. The experimental and calculated magnetic moments for a $\mathrm{Fe}_{8}$ cluster are plotted as a function of applied magnetic field for several temperatures. (a) $\theta=0$; (b) $\theta=45^{\circ}$. data, which may be due to the fact that the characteristics of the $\mathrm{Fe}_{8}$ clusters begin to change [14,15]. Similarly the isothermal magnetization data for isotropic particles have been calculated. It is found that, as a function of temperature, the magnetization decreases much faster than that for the anisotropic particle system.

By using the calculated isothermal magnetization data and Eq. (1), the values of $-\Delta S_{M}$ were calculated for a $3 \mathrm{~T}$ change in $H$ (from 0 to $3 \mathrm{~T}$ ). The calculated $-\Delta S_{M}$ data were plotted in Fig. 2 (solid lines) for the comparison with the experimental data. Excellent quantitative agreement is observed in the low temperature region. When the temperature is higher than $20 \mathrm{~K}$, the disagreement between the two sets of data begins, which may be again due to the fact that the characteristic properties of the $\mathrm{Fe}_{8}$ clusters begin to change $[14,15]$.

The values of $-\Delta S_{M}$ obtained from the calculated data for isotropic clusters are also shown in Fig. 2. The most striking feature in Fig. 2 is that $-\Delta S_{M}(T)$ shows very different behavior when the applied magnetic field is parallel and perpendicular to the easy axis of the $\mathrm{Fe}_{8}$ clusters (anisotropic cluster) and when the anisotropy becomes zero (isotropic particles). Therefore, it is evident that the magnetic anisotropy plays a very important role in the $-\Delta S_{M}$, which is demonstrated by the experimental data and the calculation.

It is evident that the values of $-\Delta S_{M}$ for isotropic clusters are larger than that for anisotropic particles, even for $\theta=0$ at low temperatures $(<7 \mathrm{~K})$, but are smaller at higher temperatures. Of course when the temperature is high enough, all the curves in Fig. 2 will merge together. To explain the behavior of the temperature dependent $-\Delta S_{M}$ in Fig. 2, we can start with Eq. (3) and with the easiest case, $\theta=0$. When $\theta=0$, Eq. (3) can be written as $E=-D S_{z}^{2}-g \mu_{B} H S_{z}$. The probability for a particle at state $S_{z}$ is determined by $p_{S_{z}}=$ $\frac{e^{\left(D S_{z}^{2}+g \mu_{B} H S_{z}\right) / k_{b} T}}{Z}=e^{D S_{z}^{2} / k_{B} T} \frac{e^{\left(g \mu_{B} H S_{z}\right) / k_{b} T}}{Z}$. It is clearly seen that the anisotropy energy favors the large $S_{z}$ state, especially when the anisotropy constant $D$ is large. At finite temperature, the mean magnetic moment of an anisotropy cluster is certainly larger than that for an isotropic cluster. With increasing temperature more and more particles will populate to the high energy levels (low $S_{z}$ ), and the mean magnetic moment will decrease. At a relatively high temperature (or when $g \mu_{B} S H / k_{b} T \ll 1$ ), the magnetization follows the Curie law. Since the much larger magnetic anisotropy energy in the $\mathrm{Fe}_{8}$ clusters, the population at the high energy levels becomes more difficult than that in isotropic particles. This explains why the magnetization of isotropic particles decreases faster than that of the anisotropic magnetic particles with increasing temperature in the same applied magnetic field. Figure 4 shows the temperature-dependent magnetization data measured from $\mathrm{Fe}_{8}$ clusters, i.e., the anisotropic particles, with a $3 \mathrm{~T}$ field for $\theta=0$ and the magnetization data calculated for the isotropic particles. It is clearly seen that the magnetization 


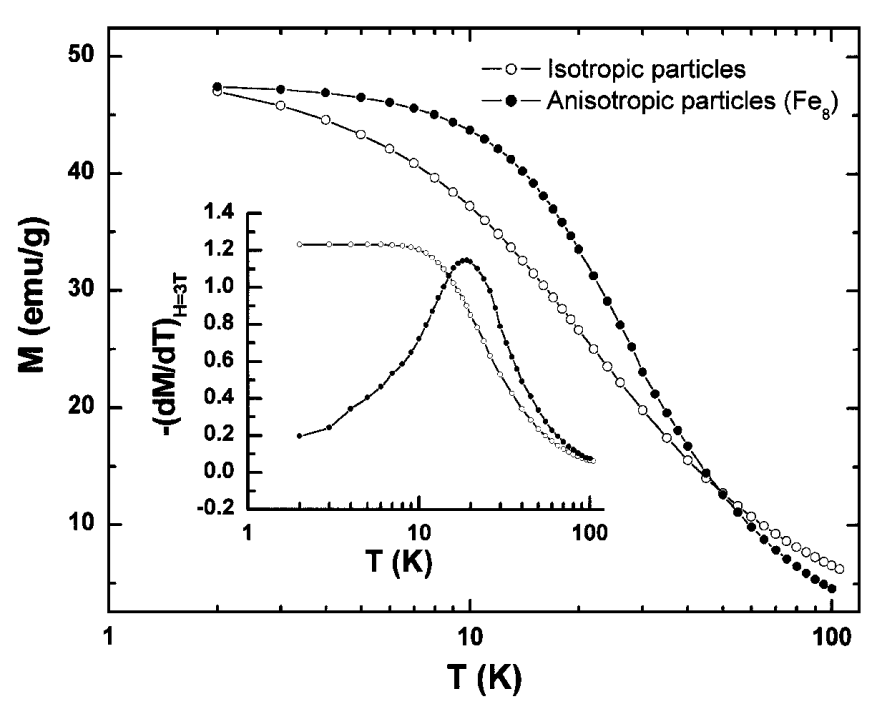

FIG. 4. The comparison of the temperature-dependent magnetization data measured from the anisotropic particles $\left(\mathrm{Fe}_{8}\right.$ clusters) (solid circles) and the calculated for isotropic clusters (open circles). The field used here is $3 \mathrm{~T}$ in both cases. The inset is the $(d M / d T) H$ versus $T$.

of the isotropic particles decreases faster than that of the anisotropic particles $\left(\mathrm{Fe}_{8}\right)$. Since the entropy change is determined by $\left(\frac{\partial M}{\partial T}\right)_{H}$, the entropy change in the isotropic particles is much larger than that in anisotropic particles in the low temperature range, but smaller at higher temperatures where the magnetization of isotropic particles is much smaller than that of anisotropic particles. The different behavior of $-\Delta S_{M}$ for the isotropic particles and the anisotropic $\mathrm{Fe}_{8}$ particles is a consequence of the different behavior of $M(T)$ and $\left(\frac{\partial M}{\partial T}\right)_{H}$ shown in Fig. 4. The behavior of $-\Delta S_{M}$ in Fig. 2 suggests that, to extend the temperature range or to increase the working temperature of the magnetic refrigerator materials, the anisotropic superparamagnetic particles are much better than the isotropic ones, especially when a magnetic field is applied parallel to the easy axis of aligned particles.

In summary, the magnetic anisotropy effects on some of the fundamental questions in the nanostructured materials have been studied using the ideal model system, $\mathrm{Fe}_{8}$ molecular crystals. The importance of this work is not only in the anisotropy effect in MCE, but also in that the magnetization (also MCE) can be calculated exactly using quantum statistics.

The authors thank Professor Ping Sheng and Professor Y.Q. Wang for useful discussions. This work was supported partially by Hong Kong RGC Grant No. HKUST6157/00E and partially by the State Key Project of Fundamental Research of China.
*Corresponding author.

Email address: phxxz@ust.hk

[1] W.F. Giauque and D.P. Macdougall, Phys. Rev. 43, 768 (1933).

[2] J. A. Barclay and W. A. Steyert, Cryogenics 22, 73 (1982).

[3] K. A. Gschneidner, Jr., and V. K. Pecharsky, J. Appl. Phys. 85, 5365 (1999).

[4] V. K. Pecharsky and K. A. Gschneidner, Phys. Rev. Lett. 78, 4494 (1997).

[5] X. X. Zhang et al., Appl. Phys. Lett. 69, 3596 (1996).

[6] F. W. Wang, X. X. Zhang, and F. X. Hu, Appl. Phys. Lett. 77, 1360 (2000); X. X. Zhang et al., Appl. Phys. Lett. 77, 3072 (2000).

[7] R. D. McMichael, R. D. Shull, L. J. Swartzendruber, L. H. Bennett, and R. E. Watson, J. Magn. Magn. Mater. 111, 29 (1992).

[8] R. D. McMichael, J. J. Ritter, and R. D. Shull, J. Appl. Phys. 73, 6946 (1993).

[9] R. D. McMichael, R. D. Shull, L. H. Bennett, C. D. Fuerst, and J. F. Herbst, Nanostruct. Mater. 2, 277 (1993).

[10] R. D. Shull, IEEE Trans. Magn. 29, 2614 (1993); R. D. Shull, AIP Conf. Proc. 273, 628 (1993).

[11] D. L. Leslie-Pelecky and R. D. Rieke, Chem. Mater. 8, 1770 (1996).

[12] J. Tejada, R. F. Ziolo, and X. X. Zhang, Chem. Mater. 8, 1784 (1996).

[13] L. H. Bennett, R. D. McMichael, R. D. Shell, L. J. Swartzendruber, and R. E. Watson, J. Appl. Phys. 73, 6507 (1993); L. H. Bennett, R. D. McMichael, H. C. Tang, and R. E. Watson, J. Appl. Phys. 75, 5493 (1994).

[14] K. Wieghardt, K. Phol, I. Jibril, and G. Huttner, Angew. Chem., Int. Ed. Engl. 23, 77 (1984).

[15] A.-L. Barra et al., Europhys. Lett. 35, 133 (1996).

[16] C. Sangregotio et al., Phys. Rev. Lett. 78, 4645 (1997).

[17] X. X. Zhang et al., J. Appl. Phys. 85, 5633 (1999).

[18] C. Delfs et al., Inorg. Chem. 32, 3099 (1993).

[19] E. del Barco et al., Europhys. Lett. 47, 722 (1999).

[20] J. R. Friedman, M. P. Sarachik, J. Tejada, and R. F. Ziolo, Phys. Rev. Lett. 76, 3830 (1996).

[21] J. M. Hernandez, X. X. Zhang, F. Luis, J. Bartolome, J. Tejada, and R. F. Ziolo, Europhys. Lett. 35, 301 (1996).

[22] E. M. Chudnovsky, Science 274, 938 (1996).

[23] L. Thomas et al., Nature (London) 383, 145 (1996); W. Wernsdorfer and R. Sessoli, Science 284, 133 (1999).

[24] F. Torres, J. M. Hernandez, X. Bohigas, and J. Tejada, Appl. Phys. Lett. 77, 3248 (2000).

[25] M. Foldeaki, R. Chahine, and T. K. Bose, J. Appl. Phys. 77, 3528 (1995).

[26] The spin Hamiltonian for a $\mathrm{Fe}_{8}$ cluster is $H=-D S_{z}^{2}+$ $E S_{x}^{2}-g \mu_{B} \vec{H} \cdot \vec{S}[15,16,19]$, where $D=0.27 \mathrm{~K}$ and $E=0.092 \mathrm{~K}$. The term $E S_{x}^{2}$ in the Hamiltonian has been neglected here, because in our sample only $c$ axes are aligned in the same direction, but the $x$ and $y$ axes are random; therefore, its effect on the determination of the magnetization is averaged out. 